\title{
Antacids may increase the appearance of white opaque substance in Helicobacter pylori-eradicated gastric epithelial neoplasia
}

\section{(ㄷ)(i)}

\author{
Authors \\ Haruhiko Takahashi ${ }^{1}$, Kenshi Yao $^{3}$, Kazunari Murakami ${ }^{4}$ \\ Institutions \\ 1 Department of Gastroenterology, Oita Red Cross \\ Hospital, Oita, Japan \\ 2 Department of Pathology, Oita Red Cross Hospital, Oita, \\ Japan \\ 3 Department of Endoscopy, Fukuoka University Chikushi \\ Hospital, Fukuoka, Japan \\ 4 Department of Gastroenterology, Faculty of Medicine, \\ Oita University, Oita, Japan
}

Kurato Wada ${ }^{1}$, Tetsuya Ueo ${ }^{1}$, Hirotoshi Yonemasu², Kazumi Togo ${ }^{1}$, Shotaro Inoue ${ }^{1}$, Hideho Honda ${ }^{1}$, Makoto Katsuta ${ }^{1}$,

submitted 18.2.2019

accepted after revision 16.5 .2019

\author{
Bibliography \\ DOI https://doi.org/10.1055/a-0961-7916 | \\ Endoscopy International Open 2019; 07: E1144-E1149 \\ (c) Georg Thieme Verlag KG Stuttgart · New York \\ eISSN 2196-9736
}

Corresponding author

Tetsuya Ueo, MD, PhD, Department of Gastroenterology, Oita Red Cross Hospital, Chiyo-machi, Oita 870-0033, Japan

Fax: +81-97-533-1207

ueo14@athena.ocn.ne.jp

\section{ABSTRACT}

Background and study aims White opaque substance (WOS) in gastric epithelial neoplasia is helpful for qualitative diagnosis of neoplasia. We hypothesized that WOS of neoplasia is strongly influenced by acid recovery after Helicobacter pylori eradication, similar to that of gastric intestinal metaplasia. The aim of this study was to investigate whether antacids increase the appearance of the WOS in H. pylori-eradicated neoplasia.

Patients and methods A total of 38 gastric epithelial neoplasias (12 adenomas and 26 adenocarcinomas) detected after $H$. pylori eradication were retrospectively evaluated. Presence or absence of WOS was evaluated by magnifying endoscopy with narrow band imaging before and after antacid administration. The $\mathrm{pH}$ of collected gastric juice was also measured. Study endpoints were (1) prevalence of WOS in the neoplasia before and after antacid administration, and the histological difference (adenoma and adenocarcinoma); and (2) relationship between the prevalence of WOS and gastric juice $\mathrm{pH}$.

Results WOS prevalence increased from $0 \%(0 / 38)$ to $44.8 \%$ (17/38) after antacid administration. WOS prevalence in adenomas was more significantly increased compared to that in adenocarcinomas $(83.3 \%$ vs $26.9 \%, P=$ $0.0077)$. Prevalence of WOS in gastric neoplasias was only observed at neutral levels of gastric juice $\mathrm{pH}$, and WOS was not observed at strong acidic levels.

Conclusions Antacid administration may increase the appearance of WOS in gastric epithelial neoplasia (especially adenomas) detected after $H$. pylori eradication with acid recovery.

\section{Introduction}

White opaque substance (WOS) on magnifying endoscopy with narrow band imaging (M-NBI) was first reported by Yao et al. as a substance in the superficial part of gastric epithelial neoplasia that obscured the subepithelial microvascular architecture [1]. Morphologic characteristics of the WOS are very helpful in distinguishing between noncancerous and cancerous lesions [1].
Moreover, we demonstrated that the nature of WOS in gastric epithelial neoplasia was an optical phenomenon caused by "accumulated lipid droplets" with fat (oil-red O) staining, immunohistochemical, and immunoelectron microscopic studies for adipophilin [2,3]. WOS in gastric epithelial neoplasia is a good marker suggesting the histological subtype of tumor (adenoma or differentiated-type carcinoma) and mucin phenotype (gastrointestinal or intestinal mucin phenotype) [2,4]. Therefore, 
presence of WOS in gastric epithelial neoplasia is very informative and a useful finding for qualitative diagnosis of the neoplasia itself.

One of the mechanisms for appearance of WOS in gastric epithelial neoplasia is believed to be "fat absorption" [2]. Ohtsu $\mathrm{K}$ et al. demonstrated that oral ingestion of external lipids promotes development of WOS in gastric epithelial neoplasia [5]. Similar "fat absorption" mechanisms have previously been reported in intestinal metaplasia of stomach. Studies of Helicobacter pylori infection-associated intestinal metaplasia of the stomach using lipid staining and light microscopy or electron microscopy showed that the epithelium in intestinal metaplasia has ability to absorb lipid droplets [6, 7]. We have more recently clarified clinical significance of WOS which signifies "absorbed lipid droplets" in intestinal metaplasia and its limitations from the perspective of intragastric acid conditions [8,9]. Interestingly, presence of WOS in intestinal metaplasia was closely associated with a state of hypochlorhydria following $H$. pylori infection, and WOS was not observed in intestinal metaplasia following acid recovery after $H$. pylori eradication $[8,9]$. This is because lipase, which plays a crucial role in lipid digestion and absorption, is inactivated in strong acidity [10]. However, after proton pump inhibitor (PPI) administration, WOS in intestinal metaplasia appeared dramatically in $45 \%$ of $\mathrm{H}$. pylori-eradicated cases following neutralization of gastric $\mathrm{pH}[8,9]$. Whether WOS in "gastric epithelial neoplasia" is similarly affected following acid recovery after $H$. pylori eradication is unknown. If that is the case, administration of antacids to neutralize intragastric acidity will facilitate appearance of WOS in gastric epithelial neoplasia after $H$. pylori eradication as well.

In this study, we focused on $\mathrm{H}$. pylori-eradicated patients because $H$. pylori eradication therapy contributes to recovery of acid secretion, not only in the intact stomach but also in the remnant gastric stump after gastrectomy $[11,12]$. The current study aimed to investigate whether antacids increase appearance of WOS in gastric epithelial neoplasia, especially detected after $H$. pylori eradication therapy.

\section{Patients and methods}

\section{Study design and participants}

At Oita Red Cross Hospital between August 2013 and June 2017, we retrospectively extracted 60 consecutive patients with gastric epithelial tumor (adenoma or adenocarcinoma) detected after $H$. pylori eradication therapy who satisfied the following inclusion criteria: (1) had undergone endoscopic submucosal dissection (ESD); (2) examined upper gastrointestinal endoscopy with M-NBI before and after PPI or potassium-competitive acid blockers ( $\mathrm{P}-\mathrm{CAB}$ ) administration; and (3) were evaluated for $\mathrm{pH}$ level of fasting gastric juice.

Subjects were excluded if they met the following criteria: (1) tumors detected within 6 months of prior $H$. pylori eradication; (2) endoscopic images insufficient for assessment; (3) history of gastric resection; or (4) tumor histologic type was undifferentiated adenocarcinoma. After 22 patients were excluded, a total of 38 patients with gastric epithelial neoplasia (12 adeno- mas and 26 adenocarcinomas) detected after $H$. pylori eradication were included in this study.

The study was approved by the institutional review board of Oita Red Cross Hospital, Oita, Japan (IRB no. 200) and conducted according to the Declaration of Helsinki. Written informed consent was obtained from all patients included in the study.

\section{Endoscopic procedures and $\mathrm{pH}$ measurements of fasting gastric juice}

All endoscopic procedures and $\mathrm{pH}$ measurements of fasting gastric juice were performed by endoscopists (K.W., T.U., H.H., S.I., and K.T.). A high-resolution magnifying upper gastrointestinal endoscope (GIF-Q240Z, Olympus Medical Systems, Tokyo, Japan) or a high-definition magnifying upper gastrointestinal endoscope (GIF-H260Z and GIF-H290Z, Olympus) and an electronic endoscopic system (EVIS LUCERA SPECTRUM, EVIS LUCERA ELITE, Olympus) were used in the current study. A soft black hood attachment (MAJ-1990 for the GIF-H260Z, MAJ1989 for the GIF-Q240Z and GIF-H290Z; Olympus) was mounted at tip of the endoscope to enable the endoscopist to fix a consistent focal distance between the tip of the endoscope and the gastric mucosa.

Endoscopic study was performed following an overnight fast of 12 hours or more. To prevent contamination from affecting accuracy of $\mathrm{pH}$ measurements, premedication (such as mucolytic or deforming agent) was not administered, except for local pharyngeal anesthesia with $8 \%$ lidocaine pump spray. Following endoscope insertion into the stomach, gastric juice pooled in the stomach was aspirated and collected endoscopically according to a previously described technique [13]. The $\mathrm{pH}$ of the collected sample was immediately measured using a 14step pH test strip (pH 1 - 14, AS ONE Co., Osaka, Japan). Following this, the observer evaluated presence or absence of WOS in the gastric neoplasia using M-NBI. The observer then recorded the results on a digital filing system (NEXUS, Fuji Film Medical Co., Tokyo, Japan). ESD was performed on the same day of the second endoscopy study.

\section{WOS definition in gastric epithelial neoplasia}

We evaluated WOS in gastric epithelial neoplasia based on findings of M-NBI before and after antacid administration. We defined WOS as "negative" or "positive" according to the proportion of WOS positivity. We labeled neoplasia as "WOS negative" when no WOS was detected in the neoplasia. WOS-positive cases were classified into the following three groups according to proportion of the WOS: a) WOS focal; WOS positivity more than $0 \%$ and up to $25 \%$, b) WOS moderate; WOS positivity more than $25 \%$ and up to $50 \%$, c) WOS diffuse; WOS positivity more than $50 \%$.

\section{Endpoints}

Endpoints of the study were: (1) prevalence of WOS in the neoplasia before and after antacid administration, and their histological difference (adenoma and adenocarcinoma); and (2) relationship between WOS prevalence and gastric juice $\mathrm{pH}$. 
- Table 1 Clinicopathological characteristics of patients divided into two groups by WOS after antacid administration.

\begin{tabular}{|l|l|l|l|}
\hline & WOS-positive & WoS-negative & Total (n=38) \\
\hline Male/female & $13 / 4$ & $15 / 6$ & $28 / 10$ \\
\hline Age, mean \pm SD (years) & $72.1 \pm 8.3$ & $73.0 \pm 8.7$ & $72.6 \pm 8.3$ \\
\hline Adenoma/Adenocarcinoma & $10 / 7$ & $2 / 19$ & $12 / 26$ \\
\hline Tumor size, mean \pm SD (mm) & $14.4 \pm 10.1$ & $11.8 \pm 7.6$ & $13.1 \pm 8.7$ \\
\hline Macroscopic type (elevated/depressed) & $9 / 8$ & $2 / 19$ & $11 / 27$ \\
\hline Tumor location (upper/middle/lower) & $1 / 9 / 7$ & $2 / 9 / 10$ & $3 / 18 / 17$ \\
\hline Depth of invasion (mucosal/submucosal) & $15 / 2$ & $15 / 6$ & $30 / 8$ \\
\hline Months after H. pylori eradication, mean \pm SD (month) & $49.6 \pm 36.6$ & $64.0 \pm 45.1$ & $57.6 \pm 41.6$ \\
\hline Days of antacid administration, mean \pm SD (day) & $24.7 \pm 15.7$ & $20.0 \pm 0.2$ & $22.1 \pm 13.0$ \\
\hline PPI/P-CAB & $14 / 3$ & $17 / 4$ & $31 / 7$ \\
\hline SD, standard deviation; PPI, proton pump inhibitor; P-CAB, potassium-competitive acid blocker & \\
\hline
\end{tabular}

\section{Statistical analysis}

Continuous variables were expressed as mean \pm standard deviation (SD). For parametric variables, Student's $t$-test was used to compare the means between two groups; otherwise, a Wilcoxon's rank-sum test was used to compare means of the groups. McNemar's test was used to compare prevalence of WOS before and after antacid administration. The chi-square test was used for comparisons of prevalence among the groups. A twosided $P<0.05$ was considered statistically significant. Statistical analyses were performed using JMP-9 software (SAS Institute, Cary, North Carolina, United States).

\section{Results}

\section{Clinicopathological characteristics of patients}

A total of 38 gastric epithelial tumors detected at least 6 months after $H$. pylori eradication were evaluated in this study. The clinicopathological features are summarized in $>$ Table 1. The male to female ratio was $28: 10$. Mean \pm SD age of the patients was $72.6 \pm 8.3$ years. Histologically, there were 12 adenomas and 26 differentiated adenocarcinomas. All adenoma cases were intestinal type from histopathological findings (H\&E staining). There were no gastric-type adenomas, so-called pyloric gland adenomas. Mean \pm SD tumor size was $13.1 \pm 8.7 \mathrm{~mm}$. Mean \pm SD duration after $H$. pylori eradication to detection of neoplasia was $57.6 \pm 41.6$ months. Mean \pm SD number of days of antacid administration was $22.1 \pm 13.0$ days. Antacids (31 PPI, 7 P-CAB) have been administered mainly for treatment of gastroesophageal reflux disease-like symptoms or for reducing inflammation and mucus adhesion to background mucosa. Between the WOS-positive and WOS-negative groups after antacid administration, there was no significant difference in sex, age, tumor size, tumor location, depth of invasion, mean months after $H$. pylori eradication, mean days of antacids administration, or PPI or P-CAB administration. A significant dif-

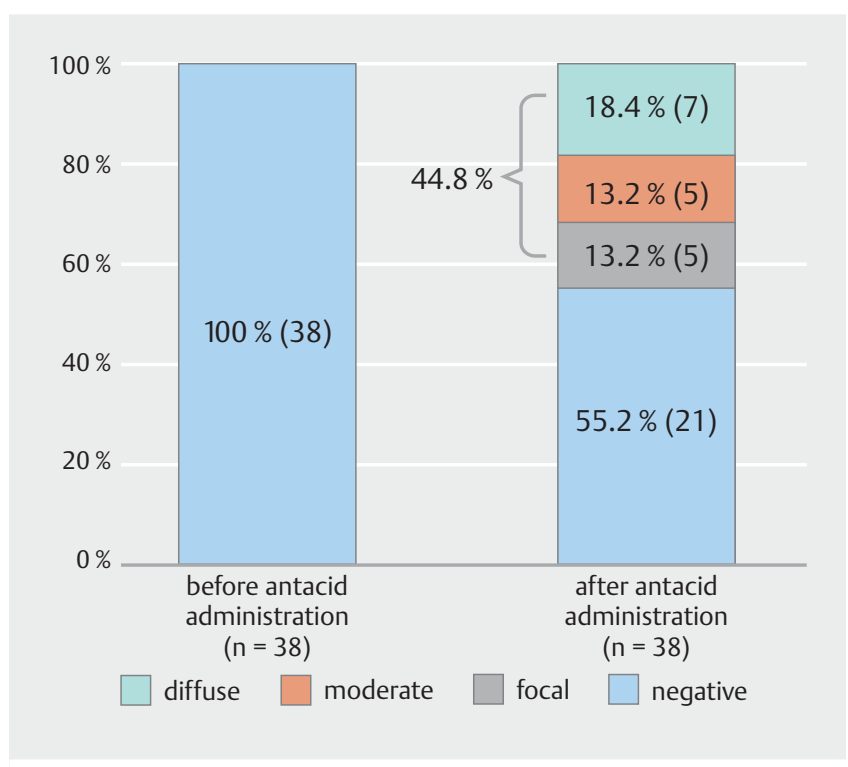

- Fig. 1 Prevalence of white opaque substance in neoplasia before and after antacid administration.

ference was observed in histological subtype $(P=0.0017)$ and macroscopic type $(P=0.0049)$.

\section{WOS prevalence in neoplasia before and after antacid administration}

Though WOS was not detected in any case $(0 \%, 0 / 38)$ before antacid administration, wOS prevalence was $44.8 \%(17 / 38)$ after antacid administration ( $\triangleright \mathbf{F i g} \mathbf{1}$ ). In the 17 WOS-positive cases, there were five WOS focal cases, five WOS moderate cases, and seven WOS diffuse cases ( $\mathbf{F i g} \mathbf{1} \mathbf{1}$ ). 


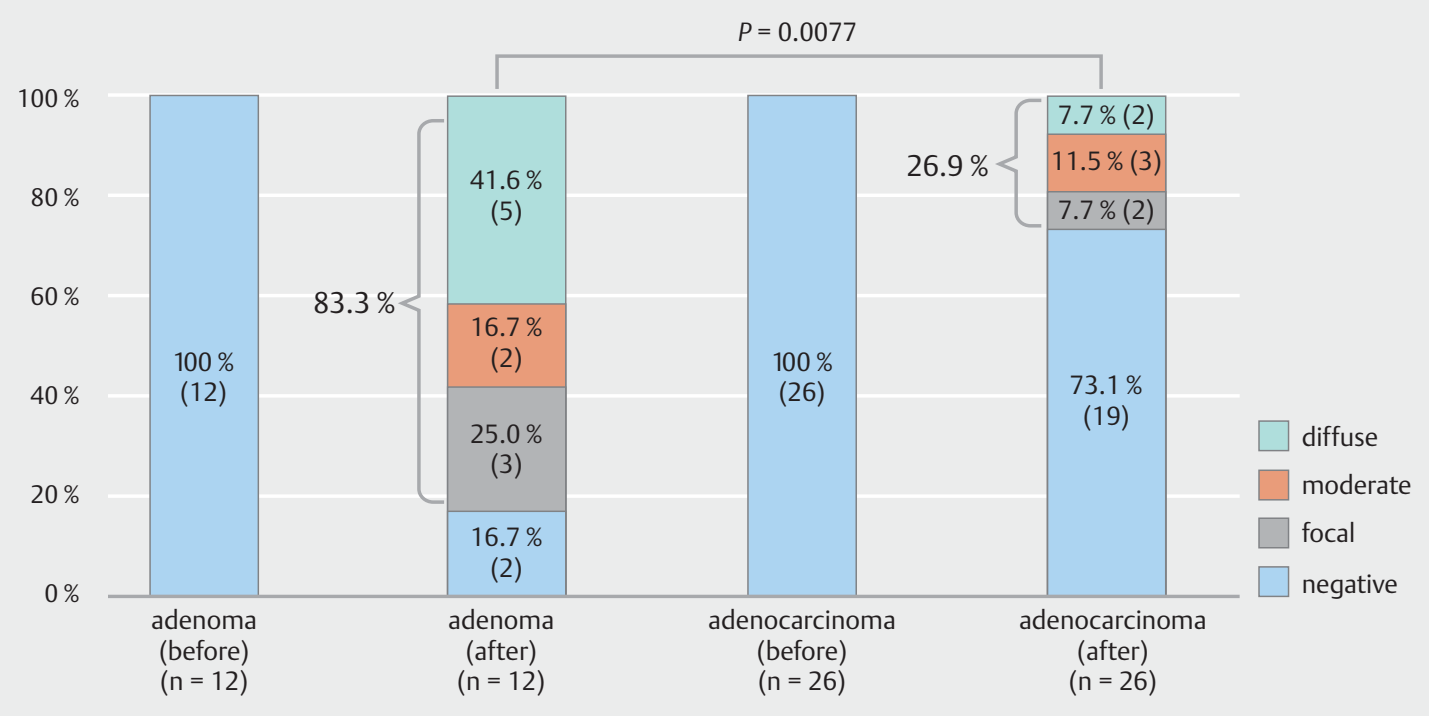

- Fig. 2 Association between histological subtype and prevalence of white opaque substance before and after antacid administration.

\section{Association between histological subtype and WOS prevalence before and after antacid administration}

After antacid administration, there was a histological difference between prevalence of WOS in adenomas and adenocarcinomas. WOS prevalence was significantly higher in patients with adenomas $(83.3 \%, 10 / 12)$ compared to that of patients with adenocarcinomas $(26.9 \%, 7 / 26)(P=0.0077)(\triangleright$ Fig. 2$)$. Among the 10 patients with WOS-positive adenomas, there were three WOS focal cases, two WOS moderate cases, and five WOS diffuse cases, while among the seven patients with WOS-positive adenocarcinomas, there were two WOS focal cases, three WOS moderate cases, and two WOS diffuse cases ( $\mathbf{F i g . 2}$ ).

\section{Relationship between prevalence of WOS and $\mathrm{pH}$ level of fasting gastric juice}

In all of the WOS-positive cases, WOS appeared after antacid administration. The $\mathrm{pH}$ levels of fasting gastric juice changed from strongly acidic $(\mathrm{pH} 1-2)$ to neutral levels $(\mathrm{pH} 7-8)$ following antacid administration. Prevalence of WOS in gastric neoplasias was only observed at neutral levels of gastric juice $\mathrm{pH}$, and WOS was not observed at strong acidic levels. ( $\triangleright$ Fig. 3, $\triangleright$ Fig. 4, and $\triangleright$ Fig. 5).

Mean \pm SD pH level after antacid administration in the WOSpositive group was $7.1 \pm 0.2$, while in the WOS-negative group it was $6.9 \pm 0.4$. There was no significant difference in $\mathrm{pH}$ level between the WOS-positive and -negative groups after antacid administration $(P=0.0571)$.

\section{Discussion}

In this study, we demonstrated utility of antacid administration for evaluation of WOS in gastric epithelial neoplasia (especially adenomas) detected in patients with acid recovery after $H$. pylori eradication. We also established a close relationship be-

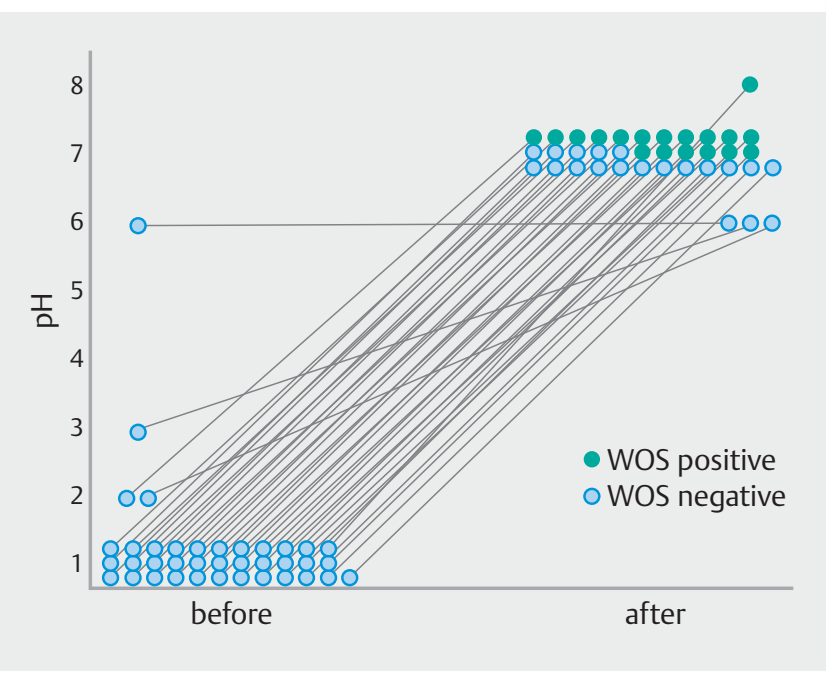

Fig. 3 Relationship between prevalence of white opaque substance and $\mathrm{pH}$ level of fasting gastric juice.

tween WOS in gastric epithelial neoplasia and intragastric acid conditions.

The study showed that presence of WOS in gastric epithelial neoplasia was only observed at a state of hypochlorhydria. In all the WOS-positive cases, WOS appearance was associated with the $\mathrm{pH}$ level of fasting gastric juice. WOS was observed at neutral $\mathrm{pH}$ levels $(\mathrm{pH} 7-8)$ but not at strongly acidic levels $(\mathrm{pH} 1-$ 2). Interestingly, these findings of WOS in gastric epithelial neoplasia were almost the same as the WOS findings in gastric intestinal metaplasia. We have more recently reported that presence of WOS in intestinal metaplasia visualized by $\mathrm{M}-\mathrm{NBI}$ was closely associated with a state of hypochlorhydria following H. pylori infection [8,9]. Among the $295 \mathrm{H}$. pylori- infected and H. pylori-eradicated cases, fasting gastric juice $\mathrm{pH}$ level was significantly higher in WOS-positive cases (mean pH 7.4 and 7.2, 


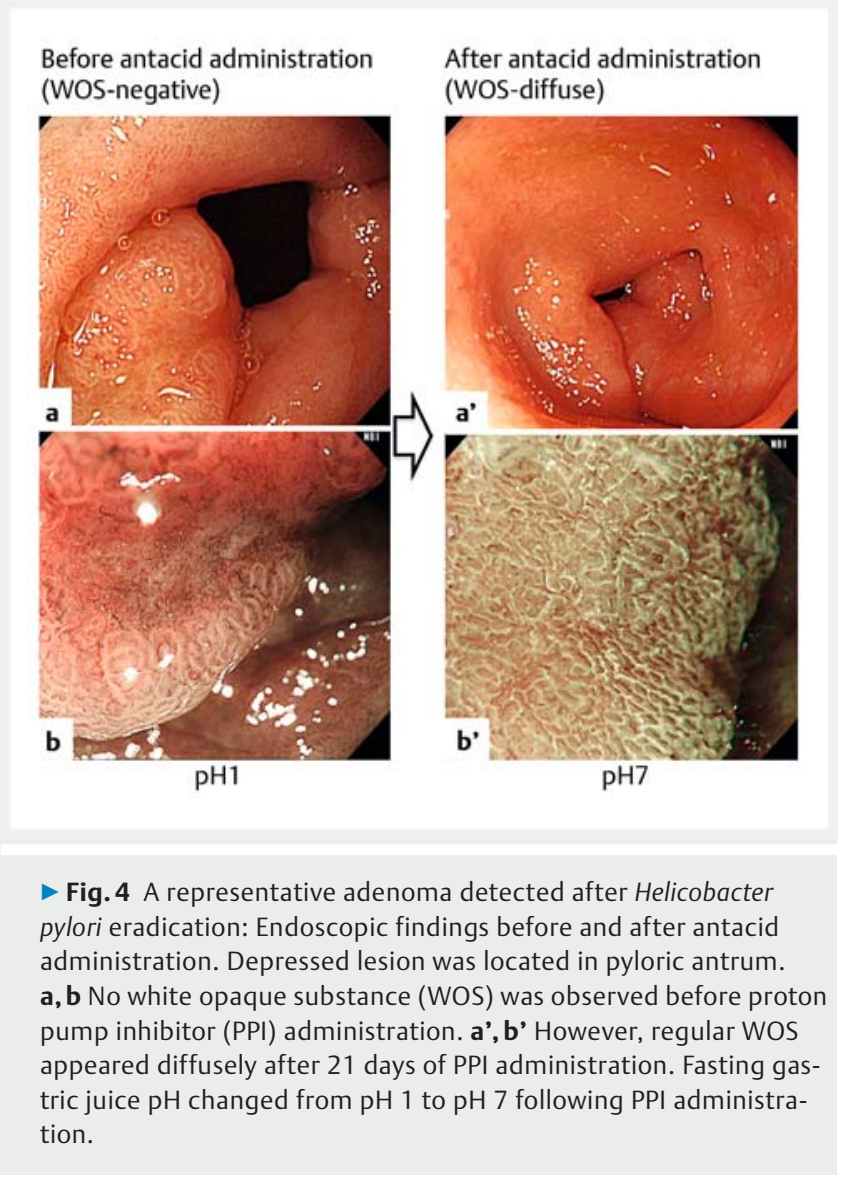

respectively) than in WOS-negative cases (mean $\mathrm{pH} 4.6$ and 1.6 , respectively) $[8,9]$. Furthermore, the relationship between presence of WOS in intestinal metaplasia and fasting gastric juice $\mathrm{pH}$ level before and after administration of PPI in $29 \mathrm{H}$. pylori-eradicated cases showed findings similar to the current study. Although we could not observe any WOS in the $29 \mathrm{H}$. pylori-eradicated cases with acid recovery, wOS dramatically appeared in $45 \%$ of these cases following neutralization of intragastric $\mathrm{pH}$ with PPI administration [8,9]. Therefore, we concluded that WOS in gastric epithelial neoplasia showed almost the same features as those in intestinal metaplasia from the perspective of intragastric acid milieu. In other words, appearance of WOS in both gastric epithelial neoplasia and intestinal metaplasia is closely related to neutralization of intragastric acid milieu, and is not a consistent phenomenon.

Morphologic characteristics of WOS are endoscopically very helpful for distinguishing between noncancerous and cancerous lesions [1]. WOS is a good marker for suggesting the histological subtype of the tumor and the mucin phenotype [2,4]. Therefore, we believe that WOS in gastric epithelial neoplasia is very informative and a useful finding for qualitative diagnosis of the neoplasia itself. However, this important WOS finding in gastric epithelial neoplasia could not be observed in $\mathrm{H}$. pylorieradicated patients with acid recovery. WOS was negative ( $0 \%$ ) in all the cases $(0 / 38)$ before antacid administration. This is a limitation of WOS. However, we established that this limitation can be overcome by using antacids. WOS appearance increased

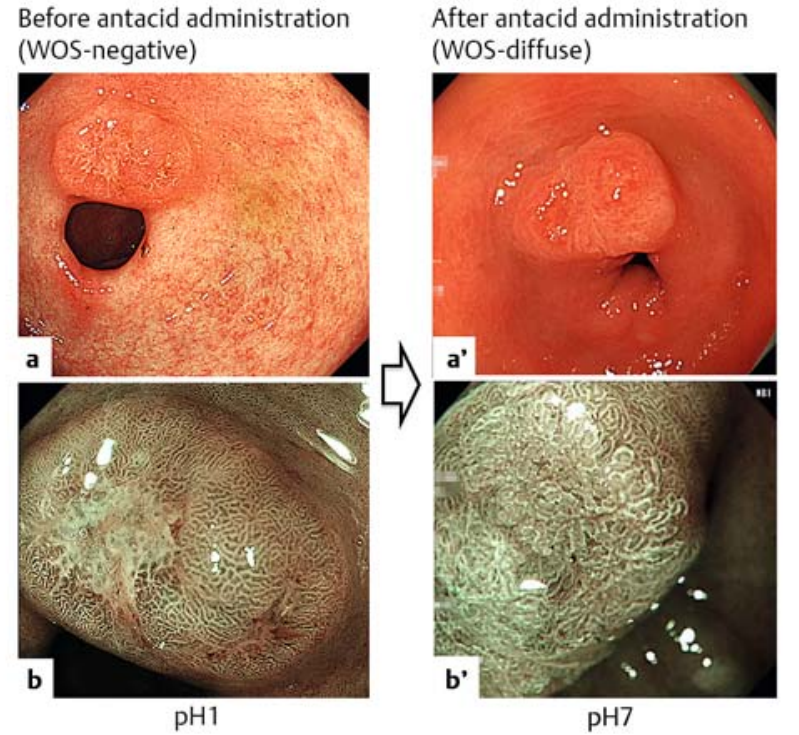

- Fig. 5 A representative adenocarcinoma detected after Helicobacter pylori eradication: Endoscopic findings before and after antacid administration. Depressed lesion was located in the pyloric antrum. a, b No white opaque substance (WOS) was observed before proton pump inhibitor (PPI) administration. a', b' However, irregular WOS appeared diffusely after 21 days of PPI administration. Fasting gastric juice $\mathrm{pH}$ changed from $\mathrm{pH} 1$ to $\mathrm{pH} 7$ following PPI administration.

to $44.8 \%(17 / 38)$ after antacid administration. That administration of an antacid (PPI or P-CAB), which causes gastric acid neutralization, may increase the appearance of WOS in gastric epithelial neoplasia in spite of the strongly recovered acidity is a significant finding. This is because incidence of gastric cancer after $H$. pylori eradication has been increasing in Japan since the Japanese government approved eradication treatment for patients with $H$. pylori-associated chronic gastritis under the National Health Insurance System beginning in February 2013. Furthermore, detection of these gastric neoplasia after eradication of $\mathrm{H}$. pylori is thought to pose difficulty with respect to boundary detection and qualitative diagnoses [14-16].

There was a histological difference in WOS prevalence after antacid administration comparing adenomas and adenocarcinomas. WOS prevalence increased more significantly in adenomas (83.3\%: 10/12) compared to that in adenocarcinomas (26.9\%: 7/26). This result was expected because it has already been reported that WOS in gastric flat elevated-type neoplasias was more frequently observed in adenoma (78\%; 14/18) compared to adenocarcinomas (43\%; 12/28) [1]. In our previous reports, we demonstrated the characteristics associated with the histologic differentiation and mucin phenotype in WOS-positive gastric neoplasia [4]. WOS was more frequently observed in adenomas (intestinal type) $(64 \%, 29 / 45)$ compared to adenocarcinomas (intestinal type or gastrointestinal type) (26\%, 22/85) [4]. We concluded that gastric neoplasia with WOS shows differentiation into a mature histological subtype with 
gastrointestinal or intestinal mucin phenotype [4]. We speculate that gastric neoplasms, especially intestinal-type adenomas, might be more strongly influenced by recovered acidity and are unable to reveal their original characteristics. We hypothesize this because we have experienced difficulty in qualitative diagnosis of gastric adenomas detected after $H$. pylori eradication [16]. Furthermore, in a previous report on endoscopic and histological reversibility of gastric adenoma after eradication of $H$. pylori, the authors concluded that $H$. pylori eradication may only mask a gastric adenoma endoscopically [17]. Though further study is necessary to clarify this issue, the appearance of WOS in gastric neoplasia (especially adenomas) following antacid administration is expected to be useful for qualitative diagnosis of those adenomas detected after $H$. pylori eradication.

This study had several limitations. First, it was retrospective and conducted at a single center. Second, we evaluated fasting gastric juice to evaluate the intragastric acid environment. The $\mathrm{pH}$ level of fasting gastric juice offers reliable information, but is not the ideal method for detection of acid secretion. Third, evaluation of mucin phenotype using immunohistochemical staining was not performed. Furthermore, the current study used both conventional PPI and a novel suppressant of gastric acid secretion called P-CAB. P-CAB reportedly has a stronger and longer anti-secretory effect than conventional PPI [18, 19]. Hence, $\mathrm{P}-\mathrm{CAB}$ may be more effective in neutralizing stomach acidity for appearance of WOS. A further well-designed prospective study using P-CAB (UMIN000032689) is currently underway to clarify these issues.

\section{Conclusion}

In conclusion, this study demonstrated that the appearance of WOS in gastric epithelial neoplasia was only observed at a state of hypochlorhydria, and WOS was not observed at strong acidic levels. Therefore, antacid administration may increase the appearance of WOS in patients with gastric epithelial neoplasia (especially adenomas) detected after $H$. pylori eradication with acid recovery.

\section{Acknowledgements}

This work was supported by the Central Research Institute for Endoscopy, Fukuoka University, Fukuoka, Kyushu, Japan.

\section{Competing interests}

\section{None}

\section{References}

[1] Yao K, Iwashita A, Tanabe $\mathrm{H}$ et al. White opaque substance within superficial elevated gastric neoplasia as visualized by magnification endoscopy with narrow-band imaging: a new optical sign for differ- entiating between adenoma and carcinoma. Gastrointest Endosc 2008; 68: $574-580$

[2] Yao K, Iwashita A, Nambu M et al. Nature of white opaque substance in gastric epithelial neoplasia as visualized by magnifying endoscopy with narrow-band imaging. Dig Endosc 201224: 419-425

[3] Ueo T, Yonemasu H, Yada N et al. White opaque substance represents an intracytoplasmic accumulation of lipid droplets: Immunohistochemical and immunoelectron microscopic investigation of 26 cases. Dig Endosc 2013; 25: 147 - 155

[4] Ueo T, Yonemasu H, Yao K et al. Histologic differentiation and mucin phenotype in white opaque substance-positive gastric neoplasia. Endosc Int Open 2015; 3 : E597-E604

[5] Ohtsu K, Yao K, Matsunaga K et al. Lipid is absorbed in the stomach by epithelial neoplasms (adenomas and early cancers): a novel functional endoscopy technique. Endosc Int Open 2015; 3: E318-E322

[6] Rubin W, Ross LL, Jeffries GH et al. Some physiologic properties of heterotopic intestinal epithelium. Its role in transporting lipid into the gastric mucosa. Lab Invest 1967; 16: $813-827$

[7] Siurala M, Tarpila S. Absorptive function of intestinal metaplasia of the stomach. Scand J Gastroenterol 1968; 3: 76-79

[8] Ueo T, Wada K, Murakami K. Clinical significance of the white opaque substance positive intestinal metaplasia evaluated by $\mathrm{H}$.pylori status. J Gastroenterol Hepatol 2017; 32: (Suppl. 03): 93

[9] Togo K, Ueo T, Yao K et al. White opaque substance visualized by magnifying narrow-band imaging is associated with intragastric acid conditions. Endosc Int Open 2018; 6: E830-E837

[10] Borgstrom B, Brockman HL. Lipases. Amsterdam: Elsevier; 1984

[11] Taguchi Y, Kaito M, Gabazza EC et al. Helicobacter pylori inhibits the secretory activity of gastric parietal cells in patients with chronic gastritis. An ultrastructural study. Scand J Gastroenterol 1997; 32: 656 663

[12] Kato S, Matsukura N, Matsuda N et al. Normalization of pH level and gastric mucosa after eradication of H.pylori in the remnant stomach. J Gastroenterol Hepatol 2008; 23: 258-261

[13] Segawa K, Nakazawa S, Tsukamoto Y et al. Estimate of gastric acid output by evaluation of fasting gastric juice collected endoscopically. Hepatogastroenterology 1991; 38: 79-82

[14] Kobayashi M, Hashimoto S, Nishikura K et al. magnifying narrow-band imaging of surface maturation in early differentiated-type gastric cancers after Helicobacter pylori eradication. J Gastroenterol 2013; 48: $1332-1342$

[15] Ito M, Tanaka S, Takata S et al. Morphological changes in human gastric tumors after eradication therapy of Helicobacter pylori in a shortterm follow up. Aliment Pharmacol 2005; 21: 559- 566

[16] Ueo T, Yonemasu H, Wada K. Attempt to improve qualitative diagnosis by administration of PPI to gastric adenoma detected after H.pylori eradication. Journal of Japanese Society of Gastroenterology 2017; 114: A252 (in Japanese)

[17] Gotoda T, Saito D, Kondo $\mathrm{H}$ et al. Endoscopic and histological reversibility of gastric adenoma after eradication of Helicobacter pylori. J Gastroenterol 1999; 34: 91 - 96

[18] Hori Y, Imanishi A, Matsukawa ] et al. 1-[5-(2-Fluorophenyl)-1-(pyridin-3-ylsulfonyl)-1H-pyrrol-3-yl]-N-methylmethanamine monofumarate (TAK-438), a novel and potent potassium-competitive acid blocker for the treatment of acid-related diseases. J Pharmacol Exp Ther 2010; 335: $231-238$

[19] Sakurai Y, Mori Y, Okamoto H et al. Acid-inhibitory effects of vonoprazan $20 \mathrm{mg}$ compared with esomeprazole $20 \mathrm{mg}$ orrabeprazole $10 \mathrm{mg}$ in healthy adult male subjects-a randomised open-labelcrossover study. Aliment Pharmacol Ther 2015; 42: 719-730 